\title{
Severe Disorders of Consciousness: The Evolution of Care
}

\section{Stacey L. Sheon JD*}

Health Law LL.M., MSOTR/L, Houston, Texas, USA

\begin{abstract}
Emergency medical treatment and intensive care are responsible for the survival of many individuals who would have otherwise died because of an injury to their brain. Consequently, an increased number of people are in vegetative and minimally conscious states. Advances in diagnostic methods, prognostic knowledge, neuroimaging, pharmacology, and proactive treatment approaches necessitate renewed consideration of patient autonomy and clinical experimentation issues for the interest of the patient and society as a whole. Care guidelines for minimally conscious states should be established and guidelines for vegetative states should be redesigned to reflect the advances in this clinical area. Furthermore, methods for accurately educating the public about disorders of consciousness and informing surrogate decision-makers need to be addressed.
\end{abstract}

\section{Introduction}

Advances in emergency medical treatment and critical care are responsible for the survival of many patients who would have otherwise died because of an injury to their brain [1,2]. Consequently, an increased number of people are in vegetative and minimally conscious states [1]. Consciousness implies that a person is aware of and attends to himself and the environment. Consciousness is maintained by impulses mediated via grey matter in the Reticular Activating System (R.A.S.). Sleep is a physiological process which is usually accompanied by reduction of impulses in the R.A.S. A conscious individual is easily arousable from sleep and has intact basic protective reflexes. Unconsciousness is different from normal sleep. Unconscious subjects are not usually arousable and quite often, there is loss of or interference with basic protective reflexes. Unconsciousness may be: Partial (semi-coma or stupor) or Complete (coma).The precise incidence and prevalence of severe disorders of consciousness are difficult to determine due in part from confusion with terminology, misdiagnosis, and disparate placements of patients [3,4].The number of individuals in the United States who sustain traumatic brain injury resulting in prolonged loss of consciousness is estimated to be 56 and 170 per one million with approximately 10,000 to 25,000 adults and 6,000 to 10,000 children receiving a diagnosis of persistent vegetative state $[4,5]$.

Management of patients with severe disorders of consciousness is a public health crisis [6].Challenges face health care professionals, family members, and caregivers who are involved in the care of this population. Differential diagnosis of vegetative states and minimally conscious states is difficult, and the rate of diagnostic error is high [7]. Misdiagnosis interferes with the development of an accurate prognosis and informed decision-making by family members or other surrogates for appropriate treatment [8]. Moreover, prolonged care for patients with a disorder of consciousness, although not invasive, consumes significant human and financial resources [9]. Most patients with a disorder of consciousness remain severely disabled needing long-term care [10].

Care guidelines do not exist for the treatment of patients in minimally conscious states [5]. The care guidelines currently available for patients in vegetative states are outdated and do not address advancements in assessment methods or proactive treatment of disorders of consciousness [5]. Restoration of consciousness and recovery of function are often desired by the patient's family members; however, access to proactive treatment is problematic because patients with severe disorders of consciousness are considered untreatable, the insurance industry in the United States does not consistently recognize proactive treatment for this population, and a significant number of treatments are still in early experimental stages $[6,10]$.

With recent advances in diagnostic methods, prognostic knowledge, neuroimaging, and proactive treatment approaches aiming for restoration and recovery, issues of patient autonomy and clinical experimentation need to be considered for the best interest of the patient and society as a whole $[8,10]$. These advances necessitate changes to care guidelines and ways of informing surrogate decisionmakers of available treatment options $[5,11]$.

\section{Disorders of Consciousness Defined}

Consciousness is the "awareness of the self and the environment" [12]. Conditions that include but are not limited to traumatic brain injury, cerebrovascular accidents, hypoxia, anoxia, epilepsy, toxin inhalation, hyper or hypo thermia, and severe hydration may cause a disorder of consciousness [13]. Disorders of consciousness include coma, vegetative state, and minimally conscious state [14].Vegetative and minimally conscious states are considered to be severe disorders of consciousness. The definition of vegetative state is the most legally relevant of the three disorders of consciousness because a number of states restrict withholding or withdrawing artificial nutrition to cases involving a person in a vegetative state [14]. Furthermore, decisions regarding the appropriateness of neurorehabilitation placement are influenced by whether the person is in a vegetative state or minimally conscious [12].

A coma is a pathological state of complete unconsciousness marked by no spontaneous eye opening and the inability to awaken a patient by application of sensory stimulation [1,4]. A patient may evolve from being in a coma into consciousness or into a vegetative state [1]. Absent complicating factors, a coma rarely lasts for more than one month $[15,16]$.

*Corresponding author: Stacey L. Sheon, Health Law LL.M., MSOTR/L, Houston, Texas, USA; E-mail: slsheon@yahoo.com

Received July 16, 2013; Accepted September 10, 2013; Published Septembe 15, 2013

Citation: Stacey L. Sheon JD (2013) Severe Disorders of Consciousness: The Evolution of Care. J Neurol Neurophysiol 4: 163. doi:10.4172/2155-9562.1000163

Copyright: $\odot 2013$ Stacey L. Sheon JD. This is an open-access article distributed under the terms of the Creative Commons Attribution License, which permits unrestricted use, distribution, and reproduction in any medium, provided the original author and source are credited. 
A vegetative state is a condition of unawareness of self and environment accompanied by sleep-wake cycles and preservation of reflexes [14]. A patient in a vegetative state "cannot think, perceive, feel, or experience [15]."Furthermore, a patient in a vegetative state does not demonstrate any evidence of "sustained, reproducible, purposeful, or voluntary behavioral responses" to stimuli [17]. If a patient remained in a vegetative state for at least one month following a brain injury, the term "persistent" was added to denote this temporal aspect of the condition [11]. The notion of "permanent" vegetative state arose in 1994 reflecting the irreversible nature of the condition if a patient remained in vegetation for three months after a non-traumatic brain injury and more than one year after a traumatic etiology [11]. Because of the confusion with terminology, the American Congress of Rehabilitation Medicine (ACRM) recommends that "persistent" and "permanent" not be used when describing a vegetative state [11]. Instead, the etiology of the vegetative state and its duration should be specified [11].

A subgroup of patients that demonstrate "minimal or inconsistent behavioral signs of consciousness" were originally described as being in a "minimally responsive state (MRS) [12]." The ACRM recommended that the term "MRS" be applied "when an 'unequivocally meaningful' behavioral response was observed following a specific command, question, or environmental prompt on at least one occasion during a period of formal assessment [12]." Later, the Aspen Workgroup recommended that the term minimally conscious state (MCS) be used instead of MRS "to emphasize the partial preservation of consciousness" in this subgroup [12]. A minimally conscious state is "a condition of severely altered consciousness in which minimal but definite behavioral evidence of self or environmental awareness is demonstrated [4].” In some instances, a minimally conscious state may be a transient one in the recovery process following a traumatic or acquired brain injury where there is widespread damage that spares brain stem and hypothalamic neurons [17]. The difference between patients in a minimally conscious state from those in a vegetative state is that the former have measurable awareness and the later do not retain it [15]. Proper management of a patient in a vegetative or minimally conscious state requires an accurate diagnosis for reaching a clinically-informed prognosis and for developing an appropriate plan of care [15].

\section{Diagnosis: Role of Standardized Assessments and Neuroimaging}

The lack of a pathological distinction between a vegetative state and a minimally conscious state creates clinical and scientific challenges [18]. Without a pathological structure to distinguish the disorders of consciousness, the diagnosis of a vegetative state or a minimally conscious state is reached by consideration of the person's clinical history, observable behaviors, and neuroimaging contributions [18].

Conventional bedside examinations of neuro-behavior are often conducted to assess the patient for spontaneous or stimulus-induced eye opening and reproducible command following $[15,19,20]$. Both eye opening and command following are viewed as evidence of awareness of the external environment and thus, consciousness [19]. There are no procedural guidelines for conducting bedside assessments of consciousness [19]. Accurate assessment of a patient's conscious awareness using a conventional bedside approach is difficult because motoric behaviors of the person may be inconsistent, small, and exhausted easily [19].Conventional bedside assessment may be further complicated by the patient's tracheotomy, arousal fluctuations, and rapidly habituating responses [21].
Despite the publication of diagnostic criteria for both vegetative and minimally conscious states, misdiagnosis rates are high [11]. In a study conducted at the Royal Hospital for Neurodisability in London, fortythree percent of the patients who were diagnosed as being in a vegetative state were actually misdiagnosed [22]. All of the misdiagnosed patients in this study were anarthric and had profound physical disabilities [22]. Sixty-five percent of these misdiagnosed individuals were blind or had severe visual impairments [22]. Misdiagnosis of a disorder of consciousness can have grave consequences [21]. The prognosis for patients in a vegetative state is less favorable than the prognosis for patients in a minimally conscious state [21]. Accurate diagnosis is critical because end-of-life decisions are influenced by whether the patient is in a vegetative or minimally conscious state [21].

The high rate of diagnostic error and the severity of the consequences of misdiagnosis illustrate the need for the use of standardized assessments with adequate validity and reliability for measuring subtle indicators of a patient's awareness and neurobehavioral functioning [21]. Research reveals that the use of validated assessment scales such as the Coma Recovery Scale - Revised (CRS-R), the Sensory Modality Assessment and Rehabilitation Technique (SMART), and the Disorders of Consciousness Scales (DOCS), to name a few, allow for the identification of small and inconsistent motor responses of awareness that may otherwise be missed $[11,15]$.

Regardless of which structured assessment method is utilized, clinicians must look for consistency of responses [11,15]. The lack of behavioral evidence of conscious awareness is not considered absolute proof of no consciousness [11]. It is important to account for confounding factors such as medical complications, infection, or medication side effects when assessing the patient [11]. Moreover, health care professionals involved with the patient's care and family members should be interviewed about whether they have observed behavior indicative of awareness [15].

In the past decade, advances in imaging techniques have contributed to the assessment of patients who have a disorder of consciousness [18]. Neuroimaging allows for information to be gathered about a patient's cognitive ability without having to rely on verbal or motor behavior [12]. Positron emission tomography (PET) scans, functional magnetic resonance imaging (fMRI), electroencephalogram (EEG), and diffusion tensor imaging (DTI) are providing information about brain function, structure, and connectivity [23]. PET scans identify disconnected pathways to the cortex because of damage to brainstem and thalamo-cortical structures [18]. PET scans, however, do not reveal the pathological difference between a vegetative state and a minimally conscious state [18]. Studies suggest that fMRI has identified residual awareness in a small number of non-responsive patients based on neuronal responses when clinicians gave commands to patients [18]. EEG literature suggests that a patient's early evoked responses to stimuli can predict a negative outcome while a late event response can predict awakening from a vegetative state [18]. By calculating the amount of diffusion and anisotropy, DTI can assess the integrity of white matter in the brain [18]. DTI may provide information about actual brain pathology in patients with a disorder of consciousness which is much needed for distinguishing vegetative from minimally conscious states [18]. At this time, neuroimaging may assist in the assessment process; however, its overall use is limited because the brain data collected by the imaging tests is not definitive [18].

\section{Prognosis for Patients with Disorders of Consciousness}

Prognosis for patients with a disorder of consciousness refers to life 
expectancy, recovery of consciousness, and recovery of function $[5,8]$. Recovery of consciousness is verified when a patient demonstrates "reliable evidence of awareness of self and the environment, consistent appearance of voluntary behavioral responses to visual and auditory stimuli, and interaction with others [5]."Prognosis should not be made based on a patient's classification as being in a vegetative state or minimally conscious state [8]. Clinicians should consider the cause of the patient's brain injury, the duration of the disorder of consciousness, the patient's biological age, the existence of co morbidities, and contributions of neuroimaging when determining the patient's prognosis [8].

According to the prognostic guideline published by the MultiSociety Task Force on Persistent Vegetative State ("Multi-Society Task Force") in 1994, the life expectancy for adult and pediatric patients in a vegetative state ranges from two to five years [5]. Causes of the shortened life expectancy include infection, generalized systemic failure, and respiratory failure [24]. Furthermore, recovery of consciousness for adults and children after twelve months is unlikely for a patient in a vegetative state that stems from a traumatic brain injury and is "exceedingly rare" after three months for a patient in a vegetative state that is the result of a non traumatic brain injury [5].

Currently, the Multi-Society Task Force's prognostic guideline is generally accepted in practice; however, two factors limit its accuracy [8]. First, a diagnosis of a minimally conscious state was not recognized before 2002 [8]. Some patients identified as being in a vegetative state may have been minimally conscious [8]. Second, the databases used by the Multi-Society Task Force may have negatively biased outcomes because many patients had life-sustaining treatment discontinued [8].

Research is showing that patients with severe disorders of consciousness "can improve after time intervals longer than those established by the Multi-Society Task Force [8]."In one study, twelve percent of patients in a vegetative state demonstrated awareness after the Multi-Society Task Force time thresholds [8]. Although these patients had a better-than-predicted prognosis, they had severe disabilities upon recovering awareness [8].

\section{Treatment of Disorders of Consciousness}

Presently, there are no care guidelines for patients in a minimally conscious state [4]. Consensus-based approaches to care consist of addressing the patient with dignity, being aware of the patient's potential to understand and perceive pain, preventing medical complications, maintaining body integrity, and establishing methods for the patient to have functional communication and interaction within his or her environment [4]. Moreover, an individual with experience in neurologic assessment of disorders of consciousness should establish the diagnosis and coordinate the clinical care of the minimally conscious patient [4].

In contrast, general care guidelines exist for managing patients in a vegetative state [5].The guidelines provide that physicians have the responsibility of discussing the patient's probabilities of recovering consciousness or remaining in a vegetative state with the patient's family members or surrogates [5]. Patients in a vegetative state "should receive appropriate medical, nursing, or home care to maintain their personal dignity and hygiene [5]." According to the established guidelines, physicians and the patient's family members "must determine appropriate levels of treatment relative to the administration and withdrawal of: 1 . Medications and other commonly ordered treatments; 2 . Supplemental oxygen and use of antibiotics; 3.Complex organ-sustaining treatments such as dialysis; 4 .
Administration of blood products; 5 . Artificial hydration and nutrition [5]."The care guidelines indicate that a do-not-resuscitate (DNR) order is appropriate for patients who are in a permanent vegetative state [5].

Existing treatment options that target improvement of a patient's level of consciousness and recovery of function such as neurorehabilitation, pharmacology, brain stimulation, and advanced care protocols are not addressed by the consensus-based approach to minimally conscious states or by the care guidelines for vegetative states [8]. Neurorehabilitation is a specialized discipline of medical practice concerned with improving chronic neurological dysfunction through "understanding of the anatomy, physiology, mechanisms of injury, and plasticity of the nervous system" and through "the development of new and more effective techniques to enhance motor control and cognitive skills [25]." Neurorehabilitation may consist of an interdisciplinary team of neurologists, other physicians, occupational therapists, physical therapists, speech therapists, music therapists, nurses, and neuropsychologists [8].Therapeutic neurorehabilitation involves splinting and proper positioning of the patient to prevent joint deformity. Neurorehabilitation also involves sensory stimulation protocols which include but are not limited to the use of sounds, music, words, visual images, touch, position changes, and weight-bearing activities to promote arousal and responsiveness [8].

Pharmacology is also used to treat patients with severe disorders of consciousness. Single pharmacological treatments are administered in an attempt to increase a patient's level of awareness and responsiveness in the environment [10]. Pharmacological interventions are based on the hypothesis that consciousness results from intact brain biochemistry, balancing the amino acid and the monoamine axes of the brain [26]. A number of different medications are being studied for their effect on a patient's arousal. Zolpidem is a fast-acting medication that impacts the amino acid axis to increase brain function following a brain injury [26]. Studies suggest that patients in vegetative as well as minimally conscious states have responded to the administration of zolpidem [26]. Zolpidem is believed to reverse neurodormancy of the brain after injury, allowing for the awakening from a vegetative state [27]. Baclofen is a GABA analogue typically used to treat spasticity after neurological injury [26]. Case reports demonstrated arousal effects from baclofen administration to patients in a vegetative state [26]. Dopaminergic agents that act on the monoamine axis, such as levodopa and bromocriptine, are documented as improving awareness in patients in a vegetative state [26]. Further, apomorphine is credited in research literature for arousing a patient from a minimally conscious state [28].

Brain stimulation is a treatment for disorders of consciousness that uses modulation of focal areas in the brain [6]. The focal areas targeted with the simulation modulate extensive networks in the brain, therefore, motor control, arousal, and cognition are impacted [6]. Alternatively, spinal cord stimulation acts on the dorsal column of the spine and provides input to the reticular system and the thalamus [6]. This input to the reticular system and thalamus results in modulation of the cerebral cortex of the brain [6]. Studies reveal that during stimulation, patients with a disorder of consciousness progressed from "no verbalization and abnormal posturing/flexion withdrawal to inconsistent verbalization and object manipulation [6]."

The complexity of providing proactive treatment for patients with severe disorders of consciousness is due, in part, to inadequate scientific evidence [4]. A review of studies on pharmacological administration, dorsal column stimulation, and deep brain stimulation reveals that documented positive results exist, however, the level of evidence was 
low [6]. Therefore, a strong recommendation concerning use or nonuse of such interventions cannot be made [6]. In order to confirm the efficacy and overall safety of the pharmacological and brain stimulation approaches, larger, controlled studies must be conducted [6].

Although the research is in its infancy, the use of an Advanced Care Protocol ("ACP") in the treatment of patients with severe disorders of consciousness yielded impressive positive findings [10].The ACP is a novel and aggressive approach to recovery of awareness and function [10]. The goal of the ACP is to "maximally normalize electrochemical balance, through multimodal neuromodulation, optimizing the brain's ability to heal and repair the injured cells and networks [10]."The ACP begins on the first day following the patient's initial assessment and is administered in three phases [10]. The first phase involves the introduction of off-label pharmaceuticals for optimizing neurotransmitter function [10]. The pharmaceutical regimen is customized for each patient [10]. The second phase incorporates median nerve stimulation "to assist in per fusing oxygen to the brain and increasing blood-brain-barrier permeability, enhancing the ability of the medications to regulate neurotransmitter stability cortically and sub cortically [10]." The third phase adds nutraceutical treatment to promote "healing, neurotransmitter production, and optimal sub cellular metabolism, and to minimize oxidative stress [10]." The treatment team using the ACP develops a nutraceutical regimen specific to each patient's needs [10].

Patients treated with the ACP also receive physical therapy, occupational therapy, speech therapy, and nursing care [10]. The team utilizes standardized clinical assessments to monitor and guide care of patients with the ACP continuing until the patient's discharge [10]. Results for patients in both vegetative and minimally conscious states who had the ACP treatment demonstrated outcomes that far surpass the American Academy of Neurology's estimates for recovery and emergence from a disorder of consciousness [10]. The American Academy of Neurology estimates that the probability of emergence from a disorder of consciousness is less than ten percent [20]. In this study, all of patients in a minimally conscious state treated with the ACP emerged to consciousness [10]. Sixty-four percent of patients in a vegetative state because of a traumatic brain injury emerged after receiving the ACP treatment [10]. Moreover, fifty-six percent of patients in a vegetative state because of a non-traumatic injury emerged with the ACP approach [10]. The ACP approach to treatment offers valuable information on management of patients with severe disorders of consciousness; however, the results of this study are preliminary and need to be confirmed with larger patient sample sizes in the future [10].

\section{Legal \& Ethical Considerations}

The concepts of coma, minimally conscious state, and vegetative state are not well understood by the lay population [29]. In fact, for centuries, even the medical community had only a crude understanding of disorders of consciousness [9]. Recent scientific advances have provided the medical community with improved knowledge of how an injury to the brain results in a severe disorder of consciousness and the probabilities about whether the patient will recover [9].

At the present time, a sizeable gap exists between medical and public views on disorders of consciousness [29]. Management of patients with a severe disorder of consciousness is a unique, expanding problem; however, it is rarely a subject of interest for the general public unless a patient has miraculously awakened from a vegetative state, or the patient is part of high profile legal battle between the family and physicians concerning withdrawal of care $[23,30]$. News coverage of such circumstances leaves a misleading impression on the public about the reality of patients with a severe disorder of consciousness [30]. A review of all major newspapers in the United States reporting on disorders of consciousness revealed that the articles emphasized cases involving conflict, controversy, violence, or accidents with skewing toward patients who had a higher probability of recovery [30]. Movies have ill-described coma and depicted patients waking up from prolonged unconsciousness with intact functioning [29].

In the very sad circumstance wherein a person sustains a brain injury that results in a severe disorder of consciousness, the family experiences extreme distress and grief [8]. Family members are faced with uncertainty, misconceptions, and ambivalence about their loved one's condition and course of care [8]. Family members tend to rely on the medical opinion of the patient's physician for treatment planning [8]. Therewith, physicians have the important responsibility of providing evidence-based information to the patient's family, free of his or her own moral beliefs and societal opinions, to enable informed decision-making to occur [8]. Given the family's grief and existing misconceptions, the physician is critical to the advancement of understanding and how management of the patient will proceed. Despite advances, the diagnostic process for a severe disorder of consciousness is difficult and development of a clear prognosis can take several months [31]. In contrast, physicians can diagnose brain death with "an extremely high rate of probability within hours to days of the original insult [31]". With brain death, the irreversible loss of all brain function is clear [31].

The absence of care guidelines for patients in a minimally conscious state and the outdated care guidelines for a vegetative state that do not address proactive treatment approaches reflect that this patient population is considered largely untreatable with regard to recovery outcomes [10]. A national survey of physicians from the American Academy of Neurology and from the American Medical Directors Association reveals "overwhelming agreement that patients in the PVS would be better off dead; that more aggressive form of medical treatment should not generally be provided; and that all medical therapy, including artificial hydration and nutrition, can be withheld in specific circumstances [32]."With regard to universal health care, the surveyed physicians also agreed with "explicit rationing of most treatments for patients in the PVS [32].”

In addition to the nature of the care guidelines and physician views on vegetative state as an untreatable condition, medical classifications of severe disorders of consciousness influence how the patient will be managed. The World Health Organization's International Statistical Classification of Diseases (9th Revision, Clinical Modification: ICD9-CM) "classify symptoms, diseases, or injuries into categories with unique codes permitting standardized epidemiological, morbidity and mortality studies, reimbursement and medical decision-making [11]."The ICD-9 code does not recognize the minimally conscious state [11]. The absence of a unique ICD-9 code for the minimally conscious state interferes with studies comparing it with the vegetative state [11]. The lacking ICD-9 code also hinders the development of prevalence statistics for disorders of consciousness [11]. Separate ICD-9-CM codes for the minimally conscious state and vegetative state which reflect the clear distinction between the two disorders is recommended in order to improve medical information retrieval and demographic studies [11].

Likewise, there are no Diagnosis-Related Groups and Current Procedural Terminology codes for severe disorders of consciousness [10]. Insurance companies in the United States do not recognize disorders of consciousness, and interventions focusing on recovery of 
function are not consistently available unless the patient's family has alternative funding or other means of accessing treatment [10].

An evolution in the care of patients with a severe disorder of consciousness is on the horizon [8].Technological advances and medical research are impacting the overall management of patients in terms of the diagnostic process, prognostic knowledge, and treatment options [8]. Neuroimaging technology is enabling the differences between the minimally conscious state and the vegetative state to be better understood [8]. Despite these advances, no proactive treatment whether it is neurorehabilitation, deep brain stimulation, spinal cord stimulation, pharmacology, or ACP has strong empirical proof of facilitating recovery of awareness and function in patients with a severe disorder of consciousness [23]. Patients with a severe disorder of consciousness are not a uniform group, and those who receive these proactive treatments essentially do so as participants in individual, clinically-based experiments [33]. The experimental nature of proactive treatments gives rise to medical-legal issues concerning how decisionmaking should occur with regard to participation in treatment as well as how to inform family members of potential treatment outcomes.

In the United States, individuals have a fundamental right to control decisions with regard to their health and bodily integrity [34]. This fundamental right is not diminished when a competent individual becomes incapacitated because of an injury to his or her brain [34]. An advance directive is a method for an individual to secure how his or her life will proceed in the event of incapacitation [33]. If a patient has not expressed autonomous preferences relevant to the situation, substitute judgment or the best interest standard applies for surrogate decision-making [9].

It is unlikely that an advance directive will designate a preference concerning participation in experimental proactive treatment, and a patient's wishes relevant to the complexities of treating a severe disorder of consciousness are often unknown [9]. Surrogate decision makers, most often the patient's family members, are left to assess whether the treatment is in the best interest of the patient [9]. Family members as surrogate decision makers are vulnerable and subject to unrealistic expectations [29]. They often seek out and consent to any and all treatments to restore their loved one's arousal and function [29].

Considering the vulnerability of the family members as surrogate decision makers, physicians who recommend proactive treatment must provide maximum disclosure that, at this time, there is no current system or guideline for such treatments and that these proactive treatments are, in fact, individual clinical experiments [8]. Physicians should clearly communicate the efficacy of the proactive treatment as well as the positive and negative possibilities for the patient [35]. Furthermore, physicians need to be cautious not to suggest therapeutic benefit if there is none [35].

Presenting the balance of the potential positive and negative possibilities of treatment to the surrogate decision makers is crucial because proactive treatments may ameliorate but do not completely eliminate the debilitating effects of severe disorders of consciousness [36]. Many individuals generally equate a patient's "greater degree of recovery of awareness" with a "greater benefit [16]."On the contrary, a patient's neurological improvement "does not necessarily translate into an improvement in the patient's psychology and quality of life [16]."

While proactive treatment may improve arousal and in some cases increase cognition and physical ability to interact within the environment, patients have severe disabilities [36]. It may be conceivable that a patient who was unaware before receiving proactive treatment now experiences emotional and physical pain following the intervention [36]. Although family members may be pleased with the patient's increased arousal and interaction within the environment, the question becomes whether or not this is truly a positive outcome for the patient [36]. With regard to proactive treatment, the fact that " $[\mathrm{r}]$ ecovering consciousness may result in harm, and recovery a greater degree of conscious may result in greater harm" must be presented to the surrogate decision makers for consideration [16].

In conclusion, care management of patients with a severe disorder of consciousness is evolving as scientific understanding of vegetative and minimally conscious states grows. Medical-legal principles such as personal autonomy and surrogate decision-making are being challenged by the neuroscientific advancements. Family members who have the task of making medical decisions for their loved one are entitled to information from the medical team that is "scientifically informed and compassionately communicated [36]."

In order for the medical community to best serve the patient, family, and society, changes need to occur. First, care guidelines for patients in a minimally conscious state need to be established and the existing care guidelines for a vegetative state need to be updated. The current care guidelines for vegetative states are not adequate for addressing the complex needs of patients with a severe disorder of consciousness in the acute phase or in the later stages of care. New guidelines for patients with a severe disorder of consciousness must address the availability of proactive treatment approaches and how these treatments should be provided. Second, in addition to developing new care guidelines for severe disorders of consciousness, a model consent form should be implemented that provides for maximum disclosure to surrogate decision-makers of the experimental nature of the proactive treatment approaches. Third, the World Health Organization should more clearly articulate its classifications for severe disorders of consciousness. With inaccurate longitudinal diagnoses and inaccurate or absent reproducible predictors of recovery will be prevented. These three recommended changes in place, physicians, healthcare professionals, and families will be better equipped to manage and protect the interests of patients with severe disorders of consciousness.

\section{References}

1. Georgiopoulos M, Katsakiori P, Kefalopoulou Z, Ellul J, Chroni E, et al. (2010) Vegetative state and minimally conscious state: a review of the therapeutic interventions. Stereotact Funct Neurosurg 88: 199-207.

2. Giacino JT, Schnakers C, Rodriguez-Moreno D, Kalmar K, Schiff N, et al (2009) Behavioral assessment in patients with disorders of consciousness: gold standard or fool's gold? Prog Brain Res 177: 33-48.

3. Beaumont JG, Kenealy PM (2005) Incidence and prevalence of the vegetative and minimally conscious states. Neuropsychol Rehabil 15: 184-189.

4. Giacino JT, Ashwal S, Childs N, Cranford R, Jennett B, et al. (2002) The minimally conscious state: definition and diagnostic criteria. Neurology 58: 349 353.

5. [No authors listed] (1995) Practice parameters: assessment and management of patients in the persistent vegetative state (summary statement). The Quality Standards Subcommittee of the American Academy of Neurology. Neurology 45: 1015-1018.

6. Oliveira L, Fregni F (2011) Pharmacological and electrical stimulation in chronic disorders of consciousness: new insights and future directions. Brain Inj 25: 315-327.

7. Julia Lechinger, Nicole Chwala-Schlegel, Robert Fellinger, Johann Donis, Gabriele Michitsch, et al. (2012) Mirroring of a simple motor behavior in Disorders of Consciousness. Clinical Neuropsychology (article in press).

8. Jox RJ, Bernat JL, Laureys S, Racine E (2012) Disorders of consciousness: responding to requests for novel diagnostic and therapeutic interventions Lancet Neurol 11: 732-738. 
Citation: Stacey L. Sheon JD (2013) Severe Disorders of Consciousness: The Evolution of Care. J Neurol Neurophysiol 4: 163. doi:10.4172/21559562.1000163

9. Racine E, Rodrigue C, Bernat JL, Riopelle R, Shemie SD (2010) Observations on the ethical and social aspects of disorders of consciousness. Can J Neurol Sci 37: 758-768

10. DeFina PA, Fellus J, Thompson JW, Eller M, Moser RS, et al. (2010) Improving outcomes of severe disorders of consciousness. Restor Neurol Neurosci 28 769-780.

11. Gosseries O, Bruno MA, Chatelle C, Vanhaudenhuyse A, Schnakers C, et al. (2011) Disorders of consciousness: what's in a name? NeuroRehabilitation 28 : $3-14$.

12. Hirschberg R, Giacino JT (2011) The vegetative and minimally conscious states: diagnosis, prognosis and treatment. Neurol Clin 29: 773-786.

13. Arciniegas DB (2011) Addressing neuropsychiatric disturbances during rehabilitation after traumatic brain injury: current and future methods. Dialogues Clin Neurosci 13: 325-345.

14. Fisher CE, Appelbaum PS (2010) Diagnosing consciousness: neuroimaging law, and the vegetative state. J Law Med Ethics 38: 374-385.

15. Bernat JL (2006) Chronic disorders of consciousness. Lancet 367: 1181-1192.

16. Walter Glannon (2011) Brain, Body, and Mind Neuroethics with a HumanFace. Oxford University Press Inc.

17. James L. Bernat (2002) Ethical Issues in the Persistent Vegetative State Patient. Neurology 283-305.

18. Fernández-Espejo D, Bekinschtein T, Monti MM, Pickard JD, Junque $C$, et al (2011) Diffusion weighted imaging distinguishes the vegetative state from the minimally conscious state. Neuroimage $54: 103-112$.

19. Bruno MA, Vanhaudenhuyse A, Thibaut A, Moonen G, Laureys S (2011) From unresponsive wakefulness to minimally conscious PLUS and functional locked-in syndromes: recent advances in our understanding of disorders of consciousness. J Neurol 258: 1373-1384.

20. Giacino JT (2005) The minimally conscious state: defining the borders of consciousness. Prog Brain Res 150: 381-395.

21. Caroline Schnakers, Audrey Vanhaudenhuyse, Joseph Giacino, Mafred Ventura, Melanie Boly, et al. (2009) Diagnostic accuracy of the vegetative and minimally conscious state: Clinical consensus versus standardized neurobehavioral assessment. BMC neurology 9: 35

22. Andrews K, Murphy L, Munday R, Littlewood C (1996) Misdiagnosis of the vegetative state: retrospective study in a rehabilitation unit. BMJ 313: 13-16.
23. Owen AM, Schiff ND, Laureys S (2009) A new era of coma and consciousness science. Prog Brain Res 177: 399-411.

24. The Multi-Society Task force on PVS (1994) Medical Aspects of the Persistent Vegetative State. N Engl J Med 330: 1572-1579.

25. Dimyan MA, Dobkin BH, Cohen LG (2008) Emerging subspecialties: neurorehabilitation: training neurologists to retrain the brain. Neurology 70 e52-54.

26. Clauss RP (2010) Neurotransmitters in coma, vegetative and minimally conscious states, pharmacological interventions. Med Hypotheses 75: 287 290 .

27. Singh R, McDonald C, Dawson K, Lewis S, Pringle AM, et al. (2008) Zolpidem in a minimally conscious state. Brain Inj 22: 103-106.

28. Fridman EA, Calvar J, Bonetto M, Gamzu E, Krimchansky BZ, et al. (2009) Fast awakening from minimally conscious state with apomorphine. Brain In 23: $172-177$

29. Racine E, Bell E (2008) Clinical and public translation of neuroimaging research in disorders of consciousness challenges current diagnostic and public understanding paradigms. Am J Bioeth 8: 13-15.

30. Wijdicks EF, Wijdicks MF (2006) Coverage of coma in headlines of US newspapers from 2001 through 2005. Mayo Clin Proc 81: 1332-1336.

31. Laureys S (2005) Science and society: death, unconsciousness and the brain Nat Rev Neurosci 6: 899-909.

32. Payne K, Taylor RM, Stocking C, Sachs GA (1996) Physicians' attitudes about the care of patients in the persistent vegetative state: a national survey. Ann Intern Med 125: 104-110.

33. JukkaVarelius (2011) Respect for Autonomy, Advance Directives, and Minimally Conscious State. Bioethics 25(9): $505-515$.

34. Ditto PH (2006) What would Terri want? On the psychological challenges of surrogate decision making. Death Stud 30: 135-148.

35. Fins JJ, Illes J, Bernat JL, Hirsch J, Laureys S, et al. (2008) Neuroimaging and disorders of consciousness: envisioning an ethical research agenda. Am J Bioeth 8: 3-12.

36. Schiff ND, Giacino JT, Fins JJ (2009) Deep brain stimulation, neuroethics, and the minimally conscious state: moving beyond proof of principle. Arch Neuro 66: 697-702. 